\title{
Effect of Balance Training on Postural Analysis of Patients suffering from Balance Disorders: A Preliminary Report
}

\author{
${ }^{1}$ Swapnil Bari, ${ }^{2}$ Somya Saxena, ${ }^{3}$ Kashif Sherwani, ${ }^{4}$ Neelesh Kumar, ${ }^{5}$ Mandeep S Dhillon
}

\begin{abstract}
Introduction: Postural stability assessment is an important task that has found widespread medical and therapeutic applications. Typically, balance measurement systems are used for this purpose. The quantitative score of postural balance is very important in determining improvement of the patients suffering from balance disorder. Postural stability of humans is highly dependent on the position of centre of pressure of the body. The analysis of sway of a human body can be successfully done by studying oscillations of its centre of pressure. The distribution of foot pressure over different regions namely medial-lateral and anteriorposterior, governs the balance of the posture in both directions.
\end{abstract}

Objective: The aim of this study is to assess quantitative changes in the postural stability of patients suffering from balance disorders after providing them biofeedback based balance training.

Materials and methods: We used Force Sensing Resistors (FSRs) to sense the magnitude of forces acting at various points of both the feet. The centre of pressure is determined using the positions of various force points and magnitude of the force acting on them. The higher capability of the body to remain at that position implies higher postural stability and a lower power of sway. The force sensors produce signal proportional to the magnitude of the foot pressure. This signal after being collected by the Data Acquisition Board is fed to the system for calculating the centre of pressure and drawing a real time graph. Different algorithms are used to determine its mean position and compare the stability. The repeated analysis shows the amount of change in patient's postural behaviour, before and after the training.

Results: Until the publication of this research work, analysis of the data of 4 patients was performed. Post intervention trials resulted in higher scores of their postural performance. Hence, favourable results were obtained showing improvement in postural stability of the patients.

Conclusion: Wider variety of ataxia patients can be chosen to perform this assessment. The system is capable of determining

\footnotetext{
${ }^{1,3}$ Postgraduate Student, ${ }^{2}$ Assistant Professor, ${ }^{4}$ Principal Scientist, ${ }^{5}$ Professor and Head

1,3,4 Department of Biomedical Instrumentation, Central Scientific Instruments Organisation, Chandigarh, India

${ }^{2,5}$ Department of Physical and Rehabilitation Medicine Postgraduate Institute of Medical Education and Research Chandigarh, India

Corresponding Author: Somya Saxena, Assistant Professor, Department of Physical and Rehabilitation Medicine, Postgraduate Institute of Medical Education and Research, Chandigarh, India, Phone: +911722756912, e-mail: somyasaxena@live.com
}

a quantitative score of improvement of the postural stability and thus can be used to assess the postural performance of patients suffering from different postural disorders.

Keywords: Balance training, Hemiplegia, Postural balance, Pre-post study, Sway analysis.

How to cite this article: Bari S, Saxena S, Sherwani K, Kumar N, Dhillon MS. Effect of Balance Training on Postural Analysis of Patients suffering from Balance Disorders: A Preliminary Report. J Postgrad Med Edu Res 2017;51(4):170-174.

Source of support: Nil

Conflict of interest: None

\section{INTRODUCTION}

Postural stability is an important aspect of human body. The distribution of the weight of body at any position governs the stability or postural balance at that point. The total pressure applied can be assumed to be concentrated at a single point known as the center of pressure (COP). The study of the oscillations of the COP in anterior posterior and lateral directions is based on sway analysis. ${ }^{1,2}$

The static position of the human body does not necessarily mean the stability of the posture. Even for a slight imbalance, the COP shifts accordingly. ${ }^{3}$ The postural balance of human body while standing depends on three major systems, namely vestibular, somatosensory, and visual system. ${ }^{4,5}$ Tilting, sliding, dizzying, vibrating, or swaying of the body results in the displacement of the COP in anterior-posterior direction or lateral direction or both of them. The lesser the displacement of the COP from the mean position, the higher the stability of the system. ${ }^{3,4}$

Postural stability assessment system does the measurement of such displacements of the COP in anteriorposterior and medial-lateral directions, thereby giving quantitative measure of the amount of sway. ${ }^{6}$ The quantitative analysis of stability or instability can be done with the help of such a system.

The basic tool of postural stability recovery is the rise in somatosensory substitutions and visual cues and enhancing the vestibular responses. The patients with temporary vestibular disorders can recover with the help of normal postural strategies, whereas severe vestibular deficits need dependence on alternate somatosensory cues. ${ }^{7}$

Thus, exercises that involve visual dependency can be performed with reduced or limited visual input but better somatosensory inputs. ${ }^{7}$ Essential postural control requires 

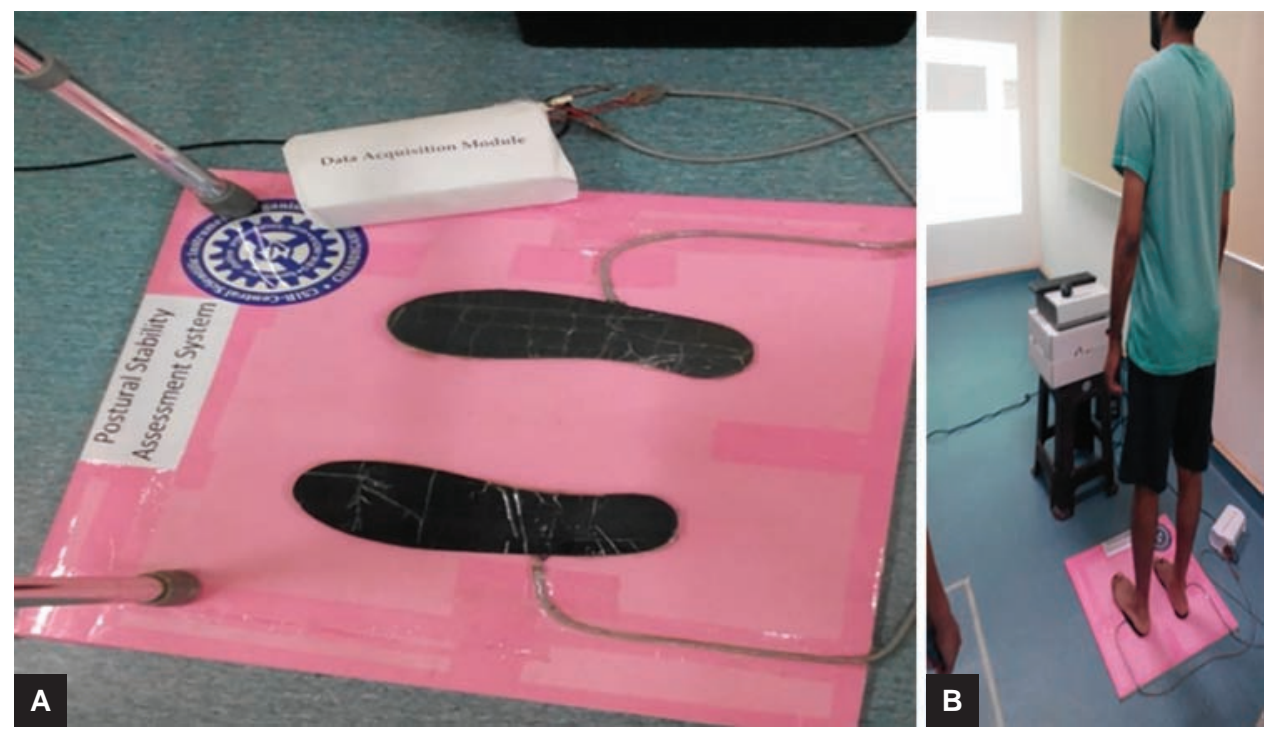

Fig. 1: (A) Image of postural stability assessment system; and (B) with subject standing on it

the central nervous system to process all the sensations and hence, control of multiple muscles simultaneously based on corresponding multisensory inputs. ${ }^{8}$

A force sensor plate is used for this purpose with sensors attached at various metatarsal points (Fig. 1). Upon detection of the force/pressure from the force sensors, the onboard processor performs mathematical calculations according to the algorithm as shown in Flow Chart 1 to determine the changes in the sway. A real-time graph can be obtained in the PC through a well-programmed channel, such as LabVIEW. A quantified analysis determines the strength of therapy needed for the rehabilitation. The complications involved in the assessment can be simplified by achieving a higher degree of biofeedback.

\section{MATERIALS AND METHODS}

\section{Study Setting}

This study was done in the Department of Physical and Rehabilitation Medicine (PRM), Postgraduate Institute of Medical Education and Research, Chandigarh, India. We used the sway analysis software incorporated in gait analysis system of the department and force sensor plates developed by Central Scientific Instruments Organisation, Chandigarh, India, for assessment of our patients.

\section{Study Design}

This was a pre-post interventional study.

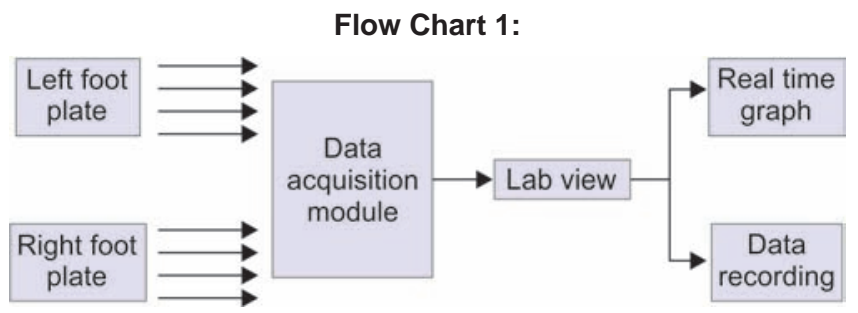

\begin{tabular}{llllll}
\multicolumn{6}{c}{ Table 1: Patient (P) characteristics } \\
\hline Subject & $\begin{array}{l}\text { Age } \\
\text { (years) }\end{array}$ & Gender & $\begin{array}{l}\text { Weight } \\
(\mathrm{kg})\end{array}$ & $\begin{array}{l}\text { Height } \\
(\mathrm{cm})\end{array}$ & Condition \\
\hline P1 & 36 & $\mathrm{M}$ & 89 & 165 & Right hemiparesis \\
P2 & 58 & $\mathrm{M}$ & 70 & 162 & Right hemiparesis \\
P3 & 13 & $\mathrm{~F}$ & 35 & 155 & Left hemiparesis \\
P4 & 18 & $\mathrm{~F}$ & 54 & 160 & Left hemiparesis \\
\hline
\end{tabular}

\section{Participants}

We included patients (Table 1) who had difficulty in walking due to neurological illness for at least 6 months. We excluded all patients who are unable to walk independently or were not willing to participate in the study.

\section{Steps for Posture Balance Analysis}

\section{Baseline (Blind)}

- Stand with both feet together for 60 seconds with eyes open

- Stand with both feet together for 60 seconds with eyes closed

\section{Intervention (Balance Training)}

- Stand on the system for 3 minutes with eyes open while maintaining the posture

- Maintain equal weight distribution on left and right foot

- Maintain the balance of both the feet in anteriorposterior direction

- Repeat the trial three times

\section{Evaluation}

Repeat both the steps of A again for the evaluation post intervention. 
The patients were made to stand on the force plates in their comfortable position and sway analysis was done for 60 seconds. The baseline readings in the first two trials with eyes open and eyes closed were recorded. Then, the patients were given three sessions of balance training. In balance training, a visual feedback was provided and patients were instructed and trained to maintain their position around $50 \%$ of the graph (midline), thus distributing equal pressure on both the feet. Similarly, the balance training was given to equally distribute pressure between ipsilateral toe and heel and the most stable posture was achieved. Finally, reevaluation of the patients was done with eyes open and closed test to compare the changes before and after balance therapy.

\section{OBSERVATIONS}

We observed that the mean displacement of $\mathrm{COP}$ was least after balance training with open eyes followed by closed eyes after training. Improvement in postural balance was observed in both steps (with open eyes and closed eyes) after training as compared with baseline values. The COP Displacement Plot for P1 to P4 are shown in Graphs 1 to 4 respectively.

For analysis, we considered anterior-posterior direction on $\mathrm{y}$-axis and lateral direction on $\mathrm{x}$-axis.

\section{RESULTS}

The mean displacement of the COP was taken in both $X$ and $Y$ directions for different trials with eyes open and eyes closed. The COP shifted to the left side in the $\mathrm{X}$ direction if the resultant pressure is higher on left side and vice versa. The COP shifted to the anterior forward in the $\mathrm{Y}$ direction if the pressure at the toe is dominant as compared with that of the heel.

\section{DISCUSSION}

Few important observations about the displacement of COP were made in our study. The magnitude of the displacement of the COP depends on the distribution of force on either side of the plane. It was observed that
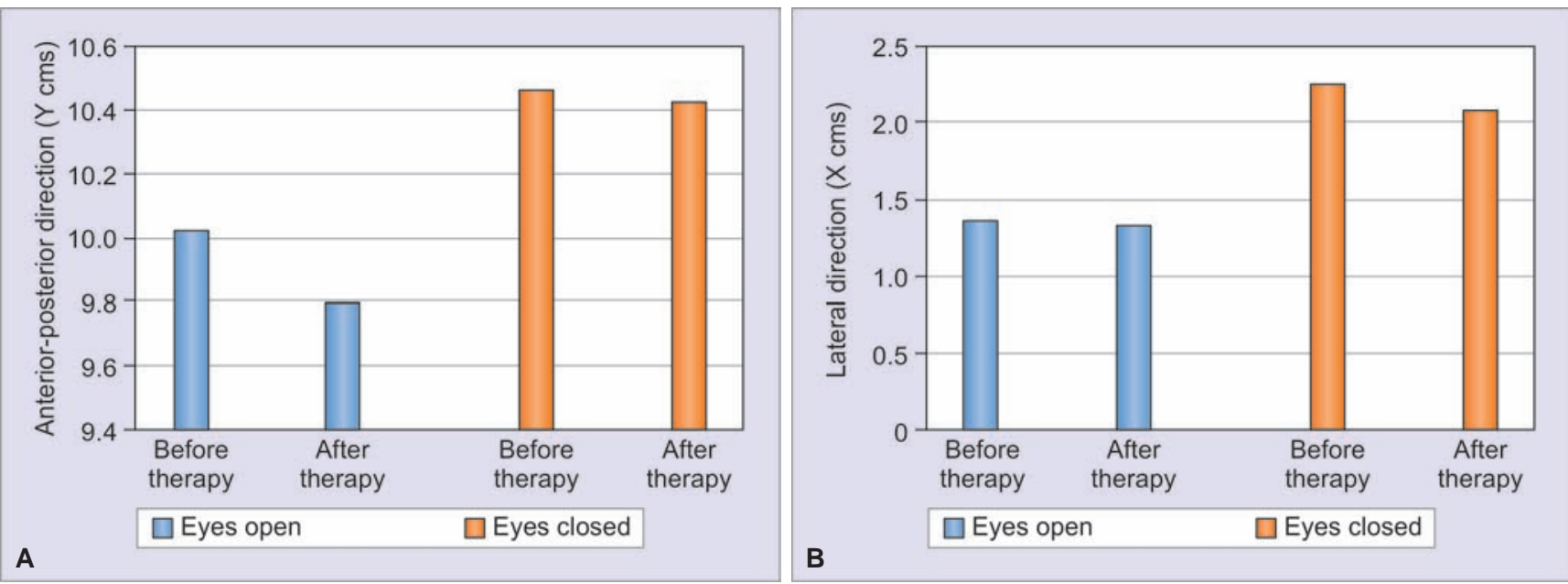

Graph 1: Mean displacement of COP of $P 1$ in $Y$ and $X$ directions
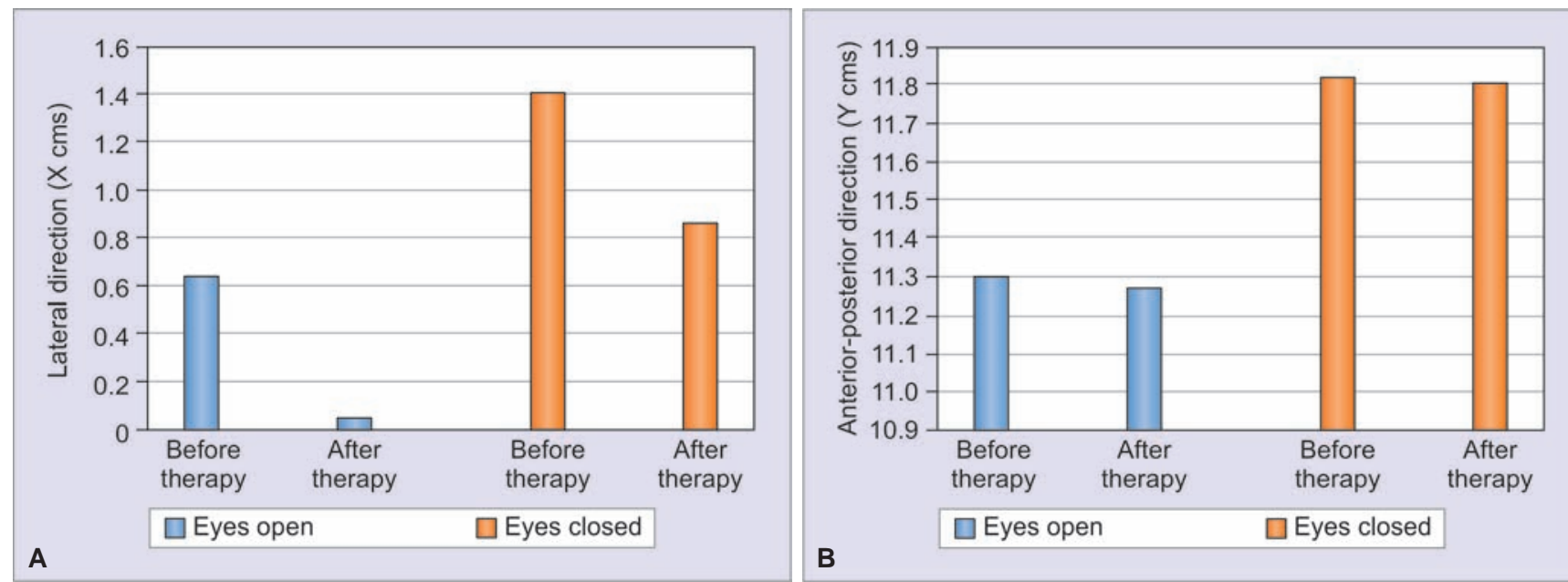

Graph 2: Mean displacement of COP of $P 2$ in $X$ and $Y$ directions 

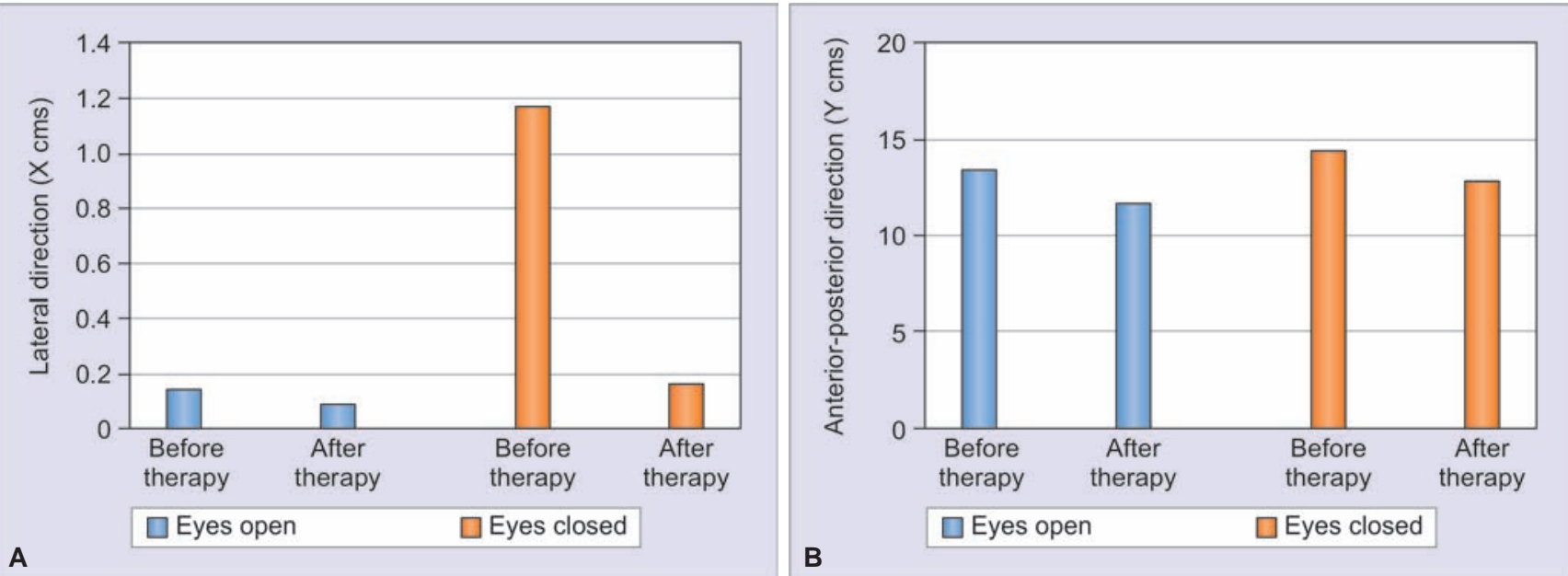

Graph 3: Mean displacement of COP of $P 3$ in $X$ and $Y$ directions
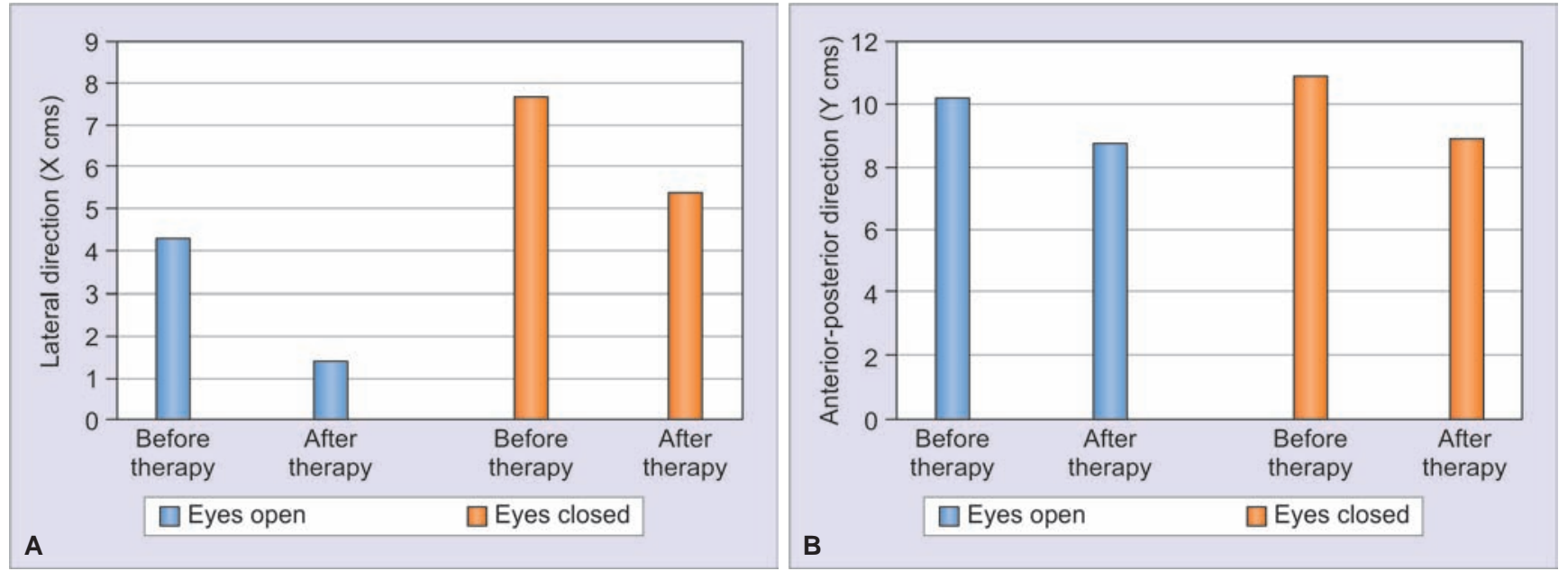

Graph 4: Mean displacement of COP of P4 in $X$ and $Y$ directions

the patients improved their balance and posture control immediately after training. There was higher displacement in COP with closed eyes, which suggests that the involvement of visual feedback is important for maintaining the postural stability. ${ }^{9}$

\section{CONCLUSION}

This is a preliminary report of four patients who were evaluated on balance assessment system for the improvement in postural control by determining the changes in displacement of the COP before and after balance training. The lower standard deviation from the mean after training (Table 2) infers the lesser displacement of the COP and thus higher stability.

It paves the way for identifying effective training modules for balance training and their use in rehabilitation settings to improve the ambulatory and functional status of the patients suffering with balance disorders. Further advanced tests and algorithms can be designed in the future to accurately monitor the sway by identifying the stability of the COP about its mean position.
Table 2: Comparison of standard deviation from mean before and after therapy

\begin{tabular}{lllllll}
\hline & & \multicolumn{2}{c}{$\begin{array}{c}\text { Std. deviation in } X \\
\text { direction }(\mathrm{cm})\end{array}$} & & \multicolumn{2}{c}{$\begin{array}{c}\text { Std. deviation in } Y \\
\text { direction }(\mathrm{cm})\end{array}$} \\
\cline { 3 - 4 } & & $\begin{array}{l}\text { Before } \\
\text { therapy }\end{array}$ & $\begin{array}{l}\text { After } \\
\text { therapy }\end{array}$ & & $\begin{array}{l}\text { Before } \\
\text { therapy }\end{array}$ & $\begin{array}{l}\text { After } \\
\text { therapy }\end{array}$ \\
\hline Patients & Eyes open & 0.272042 & 0.241836 & & 0.85405 & 0.561682 \\
P2 & Eyes closed & 0.32641 & 0.338338 & & 0.368403 & 0.302307 \\
& Eyes open & 2.393581 & 0.317409 & & 2.252183 & 0.147308 \\
& Eyes closed & 1.576525 & 1.258457 & & 0.713077 & 0.553623 \\
P3 & Eyes open & 1.078749 & 0.494888 & & 0.725202 & 0.639212 \\
& Eyes closed & 1.094055 & 0.73823 & & 0.784624 & 0.455938 \\
P4 & Eyes open & 0.511397 & 0.135609 & & 0.270095 & 0.105126 \\
& Eyes closed & 0.597992 & 0.327971 & & 0.534222 & 0.388965 \\
\hline
\end{tabular}

\section{REFERENCES}

1. Ruhe A, Fejer R, Walker B. Center of pressure excursion as a measure of balance performance in patients with non-specific low back pain compared to healthy controls: a systematic review of the literature. Eur Spine J 2011 Mar;20(3):358-368.

2. Wolff DR, Rose J, Jones VK, Bloch DA, Oehlert JW, Gamble JG. Postural balance measurements for children and adolescents. J Orthop Res 1998 Mar;16(2):271-275. 
3. Funato T, Aoi S, Tomita N, Tsuchiya K. Smooth enlargement of human standing sway by instability due to weak reaction floor and noise. R Soc Open Sci 2016 Jan;3(1):150570.

4. Lencioni T, Piscosquito G, Rabuffetti M, Bovi G, Calabrese D, Aiello A, Di Sipio E, Padua L, Diverio M, Pareyson D, et al. The influence of somatosensory and muscular deficits on postural stabilization: insights from an instrumented analysis of subjects affected by different types of Charcot-Marie-Tooth disease. Neuromuscul Disord 2015 Aug;25(8):640-645.

5. da Silva PJG, Nadal J, Infantosi AFC. Investigating the center of pressure velocity Romberg's quotient for assessing the visual role on the body sway. Rev Bras Eng Bioméd 2012 Dec;28(4):319-326.
6. Kirby RL, Price NA, MacLeod DA. The influence of foot position on standing balance. J Biomech 1987 Feb;20(4): 423-427.

7. Han BI, Song HS, Kim JS. Vestibular rehabilitation therapy: review of indications, mechanisms, and key exercises. J Clin Neurol 2011 Dec;7(4):184-196.

8. Chiba R, Takakusaki K, Ota J, Yozu A, Haga N. Human upright posture control models based on multisensory inputs; in fast and slow dynamics. Neurosci Res 2016 Mar;104:96-104.

9. Peterka RJ, Benolken MS. Role of somatosensory and vestibular cues in attenuating visually induced human postural sway. Exp Brain Res 1995 Jul;105(1):101-110. 